\title{
Quasi-Isochronous Storage Ring For Enhanced fEL Performance*
}

H. Ohgaki, D. Robin, and Y. Yamazakit

\author{
Advanced Light Source \\ Accelerator and Fusion Research Division \\ Lawrence Berkeley National Laboratory \\ University of California \\ Berkeley, CA 94720 \\ tElectrotechnical Laboratory \\ 1-1-4 Umezon \\ Tsukuba-shi, Ibaraki 305, Japan
}

August 21, 1995

Paper to be presented at the 17th International Free Electron Laser Conference and 2nd Annual FEL User Workshop, New York, NY, August 21-25, 1995.

\footnotetext{
*This work was supported by the Director, Office of Energy Research, Office of Basic Energy Sciences, Materials Sciences Division of the U.S. Department of Energy, under Contract No. DE-AC03-76SF00098
} 


\section{DISCLAIMER}

This report was prepared as an account of work sponsored by an agency of the United States Government. Neither the United States Government nor any agency thereof, nor any of their employees, make any warranty, express or implied, or assumes any legal liability or responsibility for the accuracy, completeness, or usefulness of any information, apparatus, product, or process disclosed, or represents that its use would not infringe privately owned rights. Reference herein to any specific commercial product, process, or service by trade name, trademark, manufacturer, or otherwise does not necessarily constitute or imply its endorsement, recommendation, or favoring by the United States Government or any agency thereof. The views and opinions of authors expressed herein do not necessarily state or reflect those of the United States Government or any agency thereof. 


\title{
Quasi-Isochronous Storage Ring for Enhanced FEL Performance
}

\author{
H.Ohgaki, D.Robin, and T.Yamazaki* \\ Lawrence Berkeley Laboratory, 1 Cyclotron Road, Berkeley, CA 94720 \\ *Electrotechnical Laboratory, 1-1-4 Umezono, Tsukuba-shi, Ibaraki 305
}

\section{Abstract}

A compact storage ring is designed to be used as a driver for an FEL. This ring can be operated very close to zero momentum compaction factor $(\alpha)$ to increase the electron density and thus the gain of the FEL. In order to control $\alpha$ with zero dispersion in the straight sections we use an inverted dipole located between the bending magnets and 4-families of quadrupoles. By using 3-families of sextupoles we can control the 2 transverse chromaticities and 2 nd order momentum compaction. We find that the ring has sufficient dynamic aperture for good performance.

\section{Introduction}

During that last decade several new storage ring facilities were constructed[1]. These facilities, called third generation light sources, were designed to produce small emittance electron (or positron) beams. These low emittance beams and multiple period undulators placed in long straight sections, greatly enhanced the quality of radiation emitted from insertion devices. These same qualities also make third generation light sources attractive as drivers for free-electron lasers (FEL) where the laser gain is proportional to the electron beam density and number of undulator periods [2].

Unfortunately there is a drawback to these light sources. In order to produce the small emittance beams, these light sources have a large bending radius to minimize energy dispersion. Consequently these rings are rather large -- several hundred meters in circumference. Particularly for industrial applications it would be desirable to build more compact storage rings. Because it is difficult for compact rings to produce beams with a small emittance and thus a small transverse beam size, an alternative way to increase the electron density is to decrease the electron bunch length. The bunch length in a storage ring is a function of several parameters:

$$
\sigma_{1} \propto \sqrt{\frac{L}{\rho} \frac{\gamma^{3}}{\partial V / \partial}} \alpha
$$

where $L$ is the circumference of the ring, $\rho$ is the dipole bending radius, $\gamma$ is the electron energy ( normalized to the electron rest mass), $V$ is the if voltage, and $\alpha$ is the momentum compaction factor. For a fixed beam energy and ring circumference there are several possible routes to shorter bunch lengths, but there are limitations: the bending radius can not be made very large, increasing the if voltage quickly becomes expensive and prohibitive, increasing the If frequency increases the impedance of the ring. The remaining parameter is momentum compaction. Reducing the momentum compaction factor is the approach which we have chosen to decrease the bunch length in our storage ring.

The momentum compaction in a ring is given as 


$$
\alpha=\int_{b}^{1} \frac{\eta}{\rho} d s
$$

where $\eta$ is the dispersion. There have been several attempts to reduce the momentum compaction factor using conventional storage rings and they have succeeded in shortening the bunch length to about $1 \mathrm{~mm}[3]$. In these attempts the dispersion was adjusted to be negative in part of the bending magnets. This causes the dispersion to become non-zero in the straight sections. Having non-zero dispersion is undesirable because it introduces synchrobetatron coupling in the cavities and can also degrade the quality of the radiation in the FEL. However it is possible to reduce the momentum compaction in another way by using inverted dipoles (ID, negative $\rho$ ) placed in large dispersion regions [4]. A small momentum compaction or quasi-isochronous ring (QI-ring) with inverted dipoles can realize a high peak electron current while preserving zero dispersion in the straight sections.

It should be noted that emittance in a compact QI-ring is 100 times greater than in a third generation light source. However, Deacon pointed out another important aspect of an isochronous ring[5]. An isochronous ring can generate very large FEL power because there is less longitudinal motion within the bunch.

In this paper, we describe a design for a QI-Ring with ID dedicated to StorageRing FELs to be built at the Electrotechnical Laboratory Tsukuba, Japan. The ring has two $10 \mathrm{~m}$-long dispersion free straight sections for insertion devices and a circumference of $82 \mathrm{~m}$. We first present the design of the lattice and then the results of tracking.

\section{Lattice Design}

We had to impose several requirements on the design of the ring. The main requirement is restricted space to build a new ring. We require that the circumference of the ring should be less than $85 \mathrm{~m}$. We also require that the ring have a $10 \mathrm{~m}$ long dispersion free straight section for an insertion device. The maximum energy is $1.5 \mathrm{GeV}$ and a wide tuneability in betatron functions and momentum compaction factor are also required. These requirements are based on the fact that this ring will be used not only for the FEL but also for the conventional synchrotron radiation users where it can be operated in a large $\alpha$ mode.

Figure 1 shows the overview of the QI-ring. The QI-ring consists of 4 cells, two long and two short dispersion free straight sections. The two short straight sections are used for injection and RF cavity. The long straight section are used for the insertion devices. The design work has been done by using computer codes, MAD [6], and TRACY2[7]. To reduce the circumference of the ring, we need to use a small bending radius for the bending magnets, $1.5 \mathrm{~m}$. Because we will need $3 \mathrm{~T}$ magnets to bend 1.5 $\mathrm{GeV}$ electrons in a $1.5 \mathrm{~m}$ radius, we must use superconducting bending magnets. An inverted dipole (ID) magnet is placed between the main bending magnets in a cell where the dispersion function is maximum. For a given bending angle a bending magnets contribution to the ring emittance is proportional to $\eta / \rho$. In order that the inverted dipoles not contribute very much to the emittance, a large bending radius of $10 \mathrm{~m}$ was chosen. Because of large bending radius of the $\mathrm{ID}$, a normal conducting magnet is used. The bending angle of the dipole magnets, 49 degree for main dipole and -8 degree for ID, are 
determined from the restricted circumference and from reasonably small strength of the quadrupoles.

To control the momentum compaction factor and the betatron functions, we use 4 families quadrupole magnets $(\mathrm{QF}, \mathrm{QD}, \mathrm{QFA}, \mathrm{QDA})$. The function of the quadrupole families are separated into two groups: QF and QD control the betatron tunes, QFA and QDA control the dispersion function. Consequently, we can control the momentum compaction factor keeping the dispersion function zero in the straight sections. The tunability of the momentum compaction factor is large, 0.1 to -0.1 . The lattice functions in the two cases of $\alpha$ equal 0.1 and -0.1 are shown in figure 2 . In these two cases the betatron tunes are fitted to a nominal values, $v_{x}=4.18 v_{y}=1.62$. The dispersion function is largest at the inverted dipole, and is zero in the straight section. One can see that the dispersion function remains zero in the straight section for each case. Three families of sextupole magnets $(\mathrm{S} 1, \mathrm{~S} 2, \mathrm{~S} 3)$ are used to have control the 2 transverse linear chromaticities and the second order momentum compaction[9]. We can put 3 sextupole families in the non-zero dispersion region (between the main bending and the $\mathrm{ID}$ ). The machine parameters are summarized in table 2.

Special care needs to be taken when modeling the superconducting magnets. Because of their small bending radius, the body and the fringe fields of the magnet can substantially increase the chromaticity of the ring[10]. Therefore we investigated the contribution to the overall chromaticity from both the body and fringe fields. We discovered that the body contributed very little to the chromaticity. However, there is a large contribution from the fringe fields which generate more than half of the vertical chromaticity in the ring (as shown in table 1). Therefore when modeling the bending magnets we included a nonlinear hard edge fringe field[11].

\section{Tracking Results}

There are several requirements for the size of the dynamic aperture. The first is a large enough horizontal aperture for injection. Second is a need for large energy aperture for a good Toushek life time, and third we need a large horizontal and vertical aperture for a good gas scattering lifetime. To satisfy these requirements, the ring needs a dynamic aperture which is greater than $10 \sigma_{x}, 10 \sigma_{y}$, and $10 \sigma_{e}$. We investigated the size of the dynamic aperture under a variety of situations. We looked at the size of dynamic aperture as a function of dipole fringe fields, magnet misalignments, multipole errors, and synchrotron oscillations. The tracking was performed with a sympletic integrator[12] using the computer code TRACY2. We modified TRACY2 to include a nonlinear hard edge fringe field model. The chromaticities and second order momentum compaction factor were always set to zero prior to tracking. In the simulations particles were launched at injection point with initial conditions $(\mathrm{x}, \mathrm{px}=0, \mathrm{y}, \mathrm{py}=0, \mathrm{dp} / \mathrm{p}, \tau=0)$ and tracked for 512 turns or until lost.

Figure 3 shows the effect of the fringe field on the dynamic aperture. As mentioned the first in this section, the fringe field of the superconducting bends greatly effects the dynamics of the ring considerably shrinking the dynamic aperture. However, the dynamic aperture is still large enough. We included the fringe field effects in the remainder of the simulations. 
Figure 4 shows the dynamic aperture with an RF cavity. Particles were launched off energy with a $1 \%$ energy offset $\left(\sim 10 \sigma_{e}\right)$. The dynamic aperture is reduced in horizontal plane, but not in vertical plane. However, we still have a large aperture. Figure 4 also shows the dynamic aperture with geometric misalignments. We assumed the longitudinal misalignment of $0.1 \mathrm{~mm}$ and tilt angle of $0.1 \mathrm{mrad}$ in each magnet. The dynamic aperture is not sensitive to the geometric misalignments that can be achievable. Finally, we put magnetic multipole errors into the bending and quadrupole magnets. The values of the errors are listed in table 3 taken by the reference[13]. Figure 4 shows the dynamic aperture with multipole errors on magnets: It also includes the geometric misalignments and fundamental strength errors of $0.1 \%$. The dynamic aperture largely shrinks in both planes. However, it keeps almost $10 \sigma$ in both planes. We can conclude that our design lattice has enough dynamic aperture including the effects of the fringe fields and the magnetic errors.

\section{Conclusions}

We have presented a design of a compact storage ring to be used as a driver for an FEL. This ring can be operated at a variety of different values of the momentum compaction ranging from positive to negative values. We find that the ring has ample dynamic aperture for good performance. As we lower the momentum compaction factor and thus the bunch length, we expect to be able to increase the gain of the FEL. In addition, depending upon how much we are able to lower $\alpha$ and the bunch length, this ring could produce same interesting new physics. It may be possible to generate very high powers in the FEL. Also with very short bunch lengths it will be possible to generate short pulse $\mathrm{X}$-rays and coherent infrared radiation from the bending magnets

\section{Acknowledgment}

We would like to thank A. Jackson, K.-J. Kim, and E. Forest for their helpful suggestions. This work supported by the Director, Office of Energy Research, Office of Basic Energy Science, Materials Sciences Division of the U.S. Department of Energy, under Contract No. DE-AC03-76SF00098 and the Japanese Atomic Energy Bureau.

\section{References}

[1]for instance ALS, APS, ESRF, ELETTRA.

[2]e.g. C.A.Brau, "Free-Electron Lasers", ACADEMIC PRESS INC.

[3]H.Hama, S.Takano, and. G.Isoyama, Stanford Radiation Laboratory Report No. SSRL 92/02(1992). In addition, there have been successful experimental results in Super-ACO, ESRF, and ALS.

[4]C.Pellegrini, D.Robin, Nucl. Instr. \& Meth. A301(1991)27.

A.Amiry, C.Pellegrini, E.Forest, and D.Robin, Particle Accelerators, vol-44(1994)65.

[5]D.A.G.Deacon, Physics Reports 76, No.5 (1981)349.

[6]H.Grote, F.C.Iselin, CERN/SL/90-13(1991).

[7]rewritten from TRACY by J.Bengson. For an original reference, see H.Nishimura, LBL REPORT 25236, ESG-4 (1988).

[8]R.Chasman, K.Green, E.Rowe, IEEE Trans. Nucl. Sci. 22(1975)1765.

Summarized in J.Murphy,"Synchrotron Light Source Data Book", BNL report 42333. 
[9] D.Robin, E.Forest, C.Pellegrini, and A.Amiry, Phys. Rev. E, 48(3) (1993)2149.

[10]E.Forest private communications.

[11]E.Forest, M.F.Reusch, D.L.Bruhwiler, and A.Amiry, Particle Accelerators Vol.45(1994)65.

[12]R.Ruth, IEEE Trans. Nucl. Sci. NS-30, No.4(1983)2669.

[13]ALS Conceptual Design Report, LBL PUB-5172 Rev.(1986).

Figure Captions

Fig.1 Overview of the Quasi-Isochronous Ring.

Fig. $2 \beta$ and $\eta$ functions for $\alpha=0.1$ (upper) and $\alpha=-0.1$ (lower).

Fig.3 Dynamic aperture in $x-y$ plane; the solid line displays the dynamic aperture with the fringe field effect and the dotted line displays that without the fringe field effect.

Fig.4 Dynamic aperture in $x-y$ plane; for the ideal lattice, for the off momentum particle ( $\mathrm{dp} / \mathrm{p}=1 \%$ ) with an $\mathrm{RF}$ cavity, assuming the misalignment of $0.1 \mathrm{~mm}$ for the $\mathrm{z}$-direction and of $0.1 \mathrm{mrad}$ for the tilt angle for the magnetic component, and assuming the multipole errors with the misalignment. 
Table 1

\begin{tabular}{cccc}
\hline & Bare & $\begin{array}{c}\text { including } \\
\text { body effect }\end{array}$ & $\begin{array}{c}\text { including fringe } \\
\text { fields effect }\end{array}$ \\
\hline $\begin{array}{c}\text { Natural Horizontal } \\
\text { Chromaticity }\end{array}$ & -6.124 & -5.758 & -6.124 \\
$\begin{array}{c}\text { Natural Vertical } \\
\text { Chromaticity }\end{array}$ & -6.291 & -6.234 & -11.18 \\
\hline
\end{tabular}

Table 2 Main parameters

Momentum Compaction : $\quad$ variable $(-0.1<\alpha<0.1)$

Electron Energy

$0.3-1.5 \mathrm{GeV}$ (injection at $0.3 \mathrm{GeV}$ )

Natural Emittance

$0.402 \mu-\mathrm{rad}$

Natural Energy Spread $\sigma_{e} \quad 9.91 \times 10^{-4}$

Beam size at long straight section

$$
\begin{aligned}
& \sigma_{x}: \quad 1.51 \mathrm{~mm} \\
& \sigma_{y}: \quad 1.68 \mathrm{~mm} \text { assuming full coupling, } 1.5 \mathrm{GeV}
\end{aligned}
$$

Natural Bunch Length $\quad 51 \mu \mathrm{m}\left(\mathrm{V}_{\mathrm{rf}}=1 \mathrm{MV}, \alpha=2 \times 10^{-7}\right.$, variable)

Circumference

Harmonic Number

$82.36 \mathrm{~m}$

138

RF Frequency

$502.3 \mathrm{MHz}$

Betatron Tune

Natural Chromaticity

$$
\begin{array}{ll}
\text { Horizontal } & 4.18 \\
\text { Vertical } & 1.62
\end{array}
$$

Horizontal $\quad-5.76$

Vertical -11.1

Table 3

\begin{tabular}{ccccc}
\hline \multicolumn{5}{c}{$B(x)=B \rho \sum_{n} \frac{k_{n} x^{n}}{n !}$} \\
\hline Systematic & $k I l$ & $k 2 l$ & $k 3 l$ & $k 4 l$ \\
dipoles & 0.0 & 0.05 & 0.0 & 1000.0 \\
quadrupoles & 0.0 & 0.0 & 0.0 & 0.0 \\
Random/normal & \multicolumn{2}{c}{ assumed Gaussian distribution, truncated at $2 \sigma$} \\
dipoles & $0.7 \times 10-3$ & 0.1 & 15.0 & 100.0 \\
quadrupoles & $10-3$ & 0.1 & 15.0 & 300.0 \\
\hline
\end{tabular}




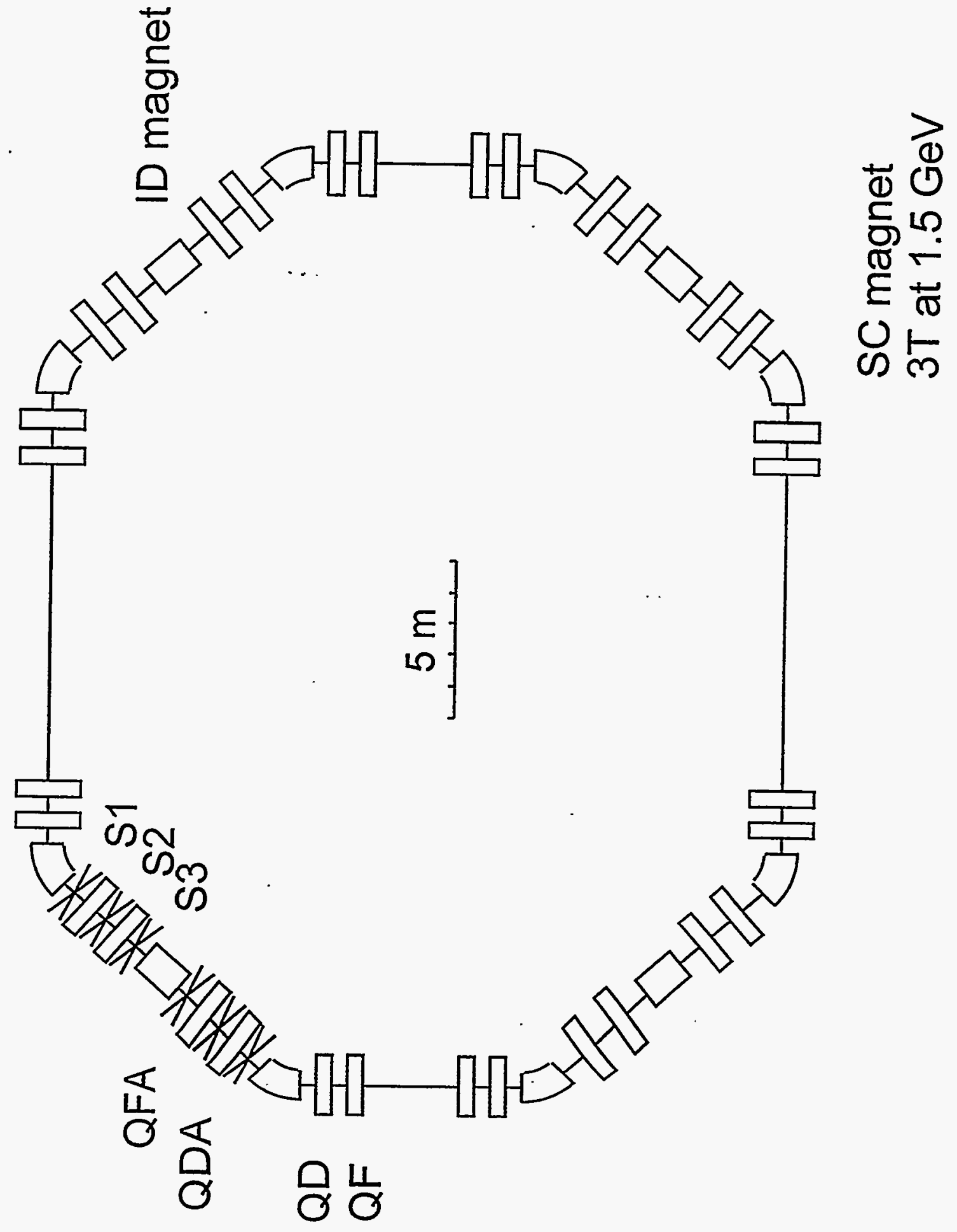




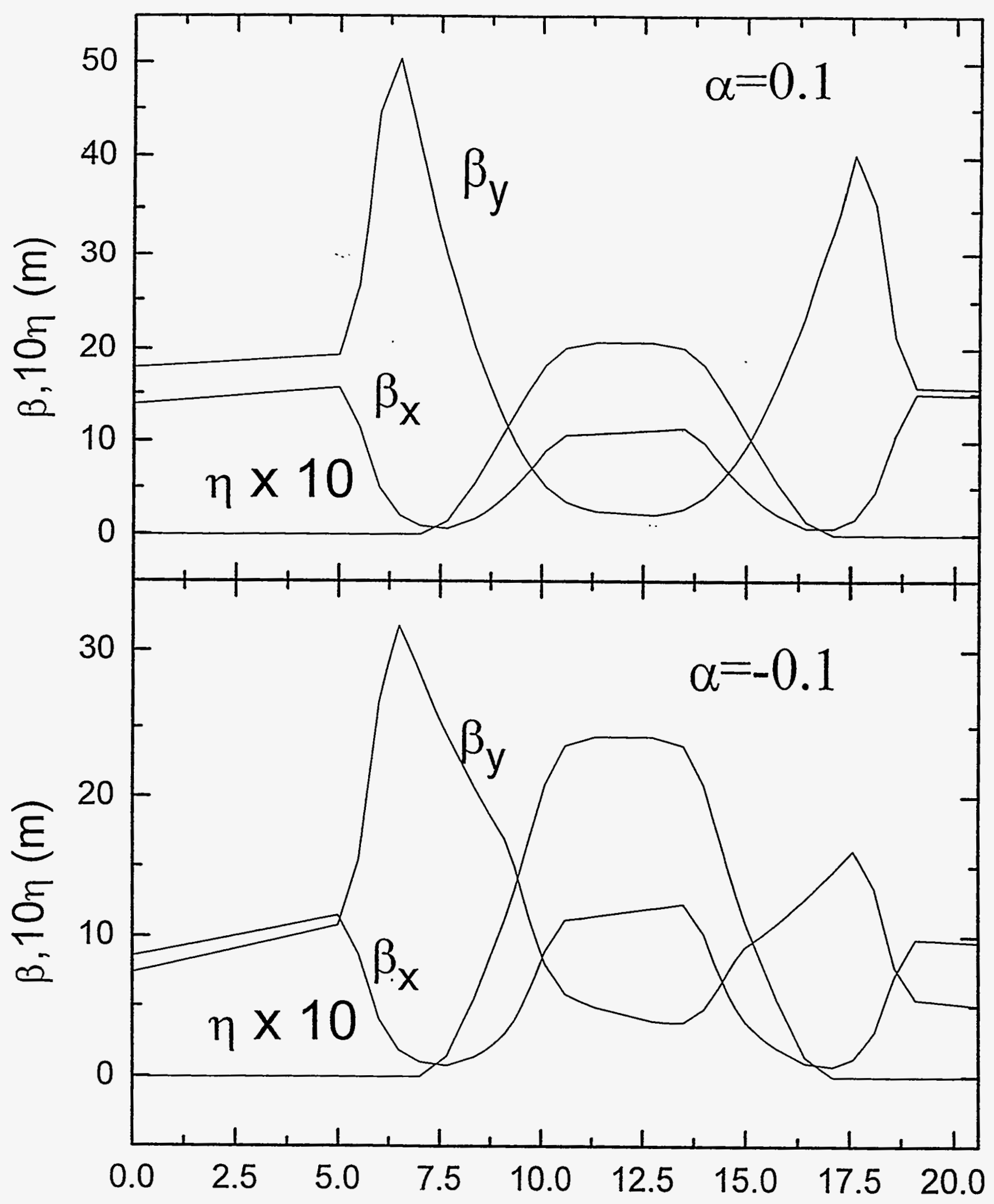




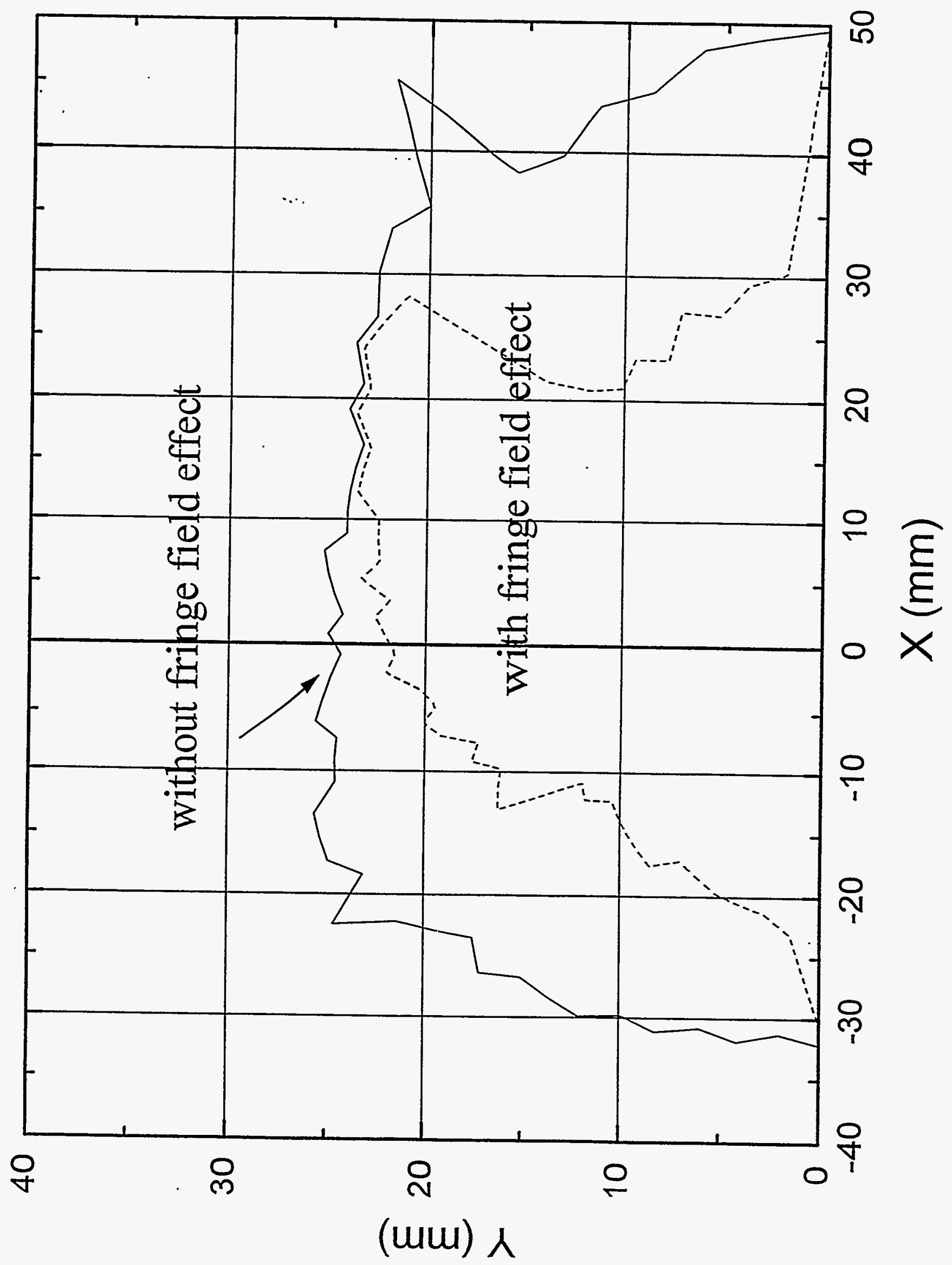




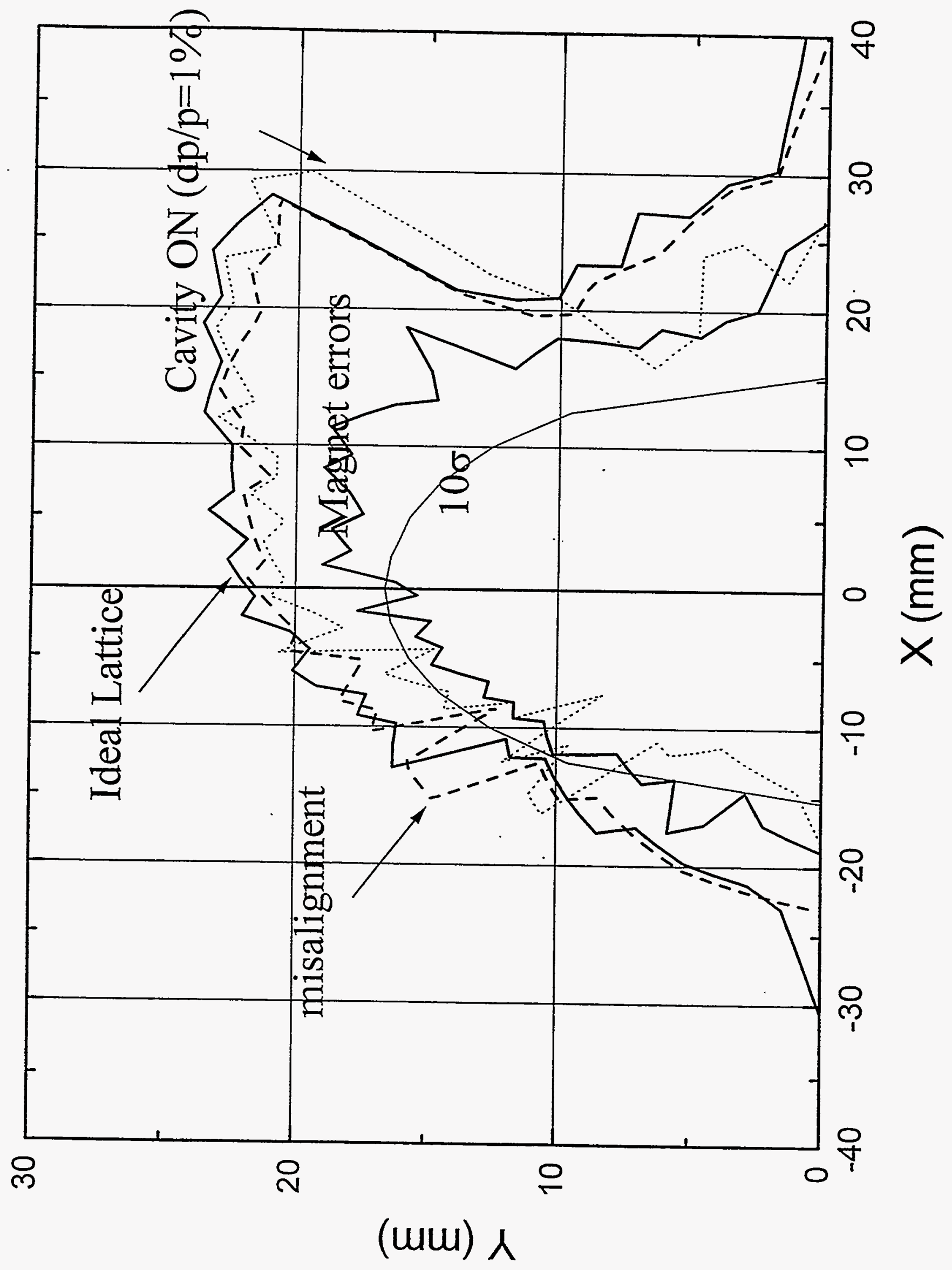

